\title{
Rivaroxaban: is it Really Need to Monitor its Anticoagulant Effect in Clinical Practice?
} Luiz Eduardo Montenegro Camanho ${ }^{\oplus}$ and Gustavo Vignoli dos Santos ${ }^{\circledR}$

Hospital Pró-Cardíaco, Rio de Janeiro, RJ - Brazil.

Editorial referring to the article: Atrial Fibrillation and use of rivaroxaban: performance of the prothrombin time/ INR as a function of time after blood collection

Oral anticoagulation (OAC) has been the cornerstone for the treatment of atrial fibrillation (AF) patients. The vitamin $\mathrm{K}$ antagonist warfarin has been considered the drug of choice in stroke prevention with proven efficacy for more than 60 years. The great inter-patient and intrapatient dose variability and need for routine International Normalized Ratio (INR), are among the main disadvantages of warfarin. ${ }^{1}$ The direct-acting oral anticoagulants (DOACs), including direct thrombin inhibitors (dabigatran) and factors Xa inhibitors (apixaban, edoxaban, and rivaroxaban), are currently the therapy of choice for preventing thromboembolic events in patients with atrial fibrillation. Unlike warfarin and other vitamin $\mathrm{K}$ antagonists, the DOACs are administered in fixed doses and do not require routine laboratory monitoring. ${ }^{2}$

Rivaroxaban is an oral inhibitor of free and clotassociated factor Xa through reversible, competitive interactions with its active site. Bioavailability following oral administration is dose-dependent, and it is highly bound to plasma proteins. ${ }^{3}$ Plasma levels peak 2 to $4 \mathrm{~h}$ following oral administration, partially excreted by the kidneys, and has a half-life depending on the dose and age. Factor Xa inhibitors variably affect prothrombin time (PT), with concentrationdependent PT prolongation. ${ }^{4}$ The sensitivity of the different assays varies widely, depending on the thromboplastin reagent, and it is recommended to check the sensitivity of PT in each institution. At therapeutic doses, rivaroxaban has a relatively weak effect on PT, but there is a more pronounced effect on supratherapeutic doses.

However, in some clinical situations, monitoring the anticoagulant effect of these drugs may be important,

\section{Keywords}

Rivaroxaban/therapeutic use; Stroke; Anticoagulants; Pulmonary Embolism; Atrial Fibrillation; Thrombosis. as in cases with increased risk of bleeding, preoperative state, breakthrough thrombosis, suspected overdose, or drug interactions, and in certain populations, including those with extremes in body weight, in the elderly and patients with renal insufficiency who are exposed to a risk of drug accumulation. ${ }^{5}$ In addition, in patients with acute ischemic stroke, the evaluation of the anticoagulant effect is extremely important before thrombolytic therapy.

In 2014, Cuker et al., ${ }^{6}$ published a systematic review and summarized evidence regarding laboratory measurement of the anticoagulant activity of dabigatran, rivaroxaban, and apixaban. ${ }^{6}$ Generally, rivaroxaban prolonged PT in a concentration-dependent, and assay results vary markedly with different thromboplastin reagents. A normal PT does not rule out the presence of clinically significant below or within on-therapy rivaroxaban concentrations; however, a prolonged PT qualitatively indicates the drug's presence. The APTT (activated partial thromboplastin time) is not suitable for measuring rivaroxaban due to the nonlinear relationship with rivaroxaban concentration, poor sensitivity, and significant variability between reagents. Inter-assay variability was reduced by the use of an international sensitivity index (ISI) specific for rivaroxaban, but not by conversion to an INR used for monitoring VKA therapy. ${ }^{7}$

In this issue of the International Journal of Cardiovascular Sciences, Duarte et al., ${ }^{8}$ evaluated 34 patients with AF in use of Rivaroxaban by using PT/INR, distributed into a subgroup with blood collection time of $12 \mathrm{~h}(\mathrm{n}=7)$ and $>12 \mathrm{~h}$ after the last drug intake $(\mathrm{n}=27) .8$ $\mathrm{PT}$, in seconds, was considerably higher in patients whose Rivaroxaban administration was $\leq 12 \mathrm{~h}$, suggesting that the test was sensitive to detect the anticoagulant effect. As a consequence, prothrombin activity, evaluated in terms of the median and interquartile range was lower

Mailing Address: Luiz Eduardo Camanho

Rua General Polidoro, 192. Postal Code: 22280-003, Rio de Janeiro, RJ - Brazil.

E-mail: lecamanho@globo.com

DOI: https://doi.org/10.36660/ijcs.20200373 
in this group. INR also appeared to be different between subgroups and was significantly higher in the $\leq 12 \mathrm{~h}$ subgroup, although this ratio is not considered a reliable mode of expression in the context of Rivaroxaban use. In conclusion, the authors suggested that, in contrast to warfarin, knowledge of the time interval between drug administration and blood collection in patients using Rivaroxaban is essential to interpret a laboratory test that evaluates hemostasis, particularly PT and its derivatives.

In 2018, Woodruff et al., ${ }^{9}$ investigated the association between prothrombin times and bleeding in hospitalized patients receiving rivaroxaban. ${ }^{9}$ In their single-center retrospective cohort study, adult patients who had the PT measured within $24 \mathrm{~h}$ of rivaroxaban administration were identified and a multivariate logistic regression model was used to quantify the association between PT and bleeding events. In total, 199 patients were identified, of which 41 experienced a bleeding event; patients with a PT $\geq 30 \mathrm{~s}$ were more likely to have experienced a bleeding event, than those with a PT $<30$ s. This study, however, does not report the exact time after the dose that the PT was measured, so it is difficult to know if like is being compared to like (in terms of time after dose) and therefore, it is again difficult to draw any firm conclusions from this data.

The results of the present study should be analyzed carefully, due to the very small number of patients and the inability to draw an exact conclusion. The question that should be answered is if it is really needed to monitor the DOACs anticoagulant effect in clinical practice. What is the clinical relevance of this approach in daily practice?

\section{References}

1. Patel JP, Byrne RA, Patel RK, Arya R. Progress in the monitoring of direct oral anticoagulant therapy. Br J Haematol. 2019;184(6):912-24.

2. Gosselin RC, Adcock DM, Bates SM, Douxfils J, Favaloro EJ, Gouin-Thibault I, et al. International Council for Standardization in Haematology (ICSH) Recommendations for Laboratory Measurement of Direct Oral Anticoagulants. Thromb Haemost. 2018;118(3):437-50.

3. Mueck W, Stampfuss J, Kubitza D, Becka M. Clinical pharmacokinetic and pharmacodynamic profile of rivaroxaban. Clin Pharmacokinet. 2014;53(1):1-16.

4. Conway SE, Hwang AY, Ponte CD, Gums JG. Laboratory and Clinical Monitoring of Direct Acting Oral Anticoagulants: What Clinicians Need to Know. Pharmacotherapy. 2017;37(2):236-48.

5. Samama MM, Contant G, Spiro TE, Perzborn E, Flem LL, Guinet C, et al. Evaluation of the prothrombin time for measuring rivaroxaban plasma concentrations using calibrators and controls: results of a multicenter field trial. Clin Appl Thromb Hemost. 2012;18(2):150-8.
The truth is, not many quality real-world studies that assessed DOAC plasma concentrations and outcomes have been published to date.

In order to answer these questions, Rottenstreich et al. have shared their experience of DOAC drug level monitoring in clinical patient management. They aimed to describe the real-life utilization of DOAC levels in practice and its effect on clinical management. ${ }^{10}$ They reviewed the data between 2013 and 2017 at their institute in Israel, with 212 patients undergoing 292 DOAC measurements. From the requests made, $82.5 \%$ were for selected clinical circumstances, e.g., bleeding or breakthrough thrombosis. The majority of patients $(71.9 \%)$ had concentrations in the expected range. Where concentrations were higher than expected, multivariate analysis revealed that older age, lower glomerular filtration rate, and lower body mass are significant determinants for these measurements. The authors concluded that whilst no benefit of routine monitoring was observed, drug level measurement has an important role in selected circumstances. Future studies are warranted to establish associations between drug levels and outcomes, and better delineate the role of DOAC monitoring.

In the current scenario, the role of monitoring the anticoagulant effect of DOACs is not yet well-defined, but we know that it can be useful in specific situations, where the risk of bleeding is extremely high or when there is an indication for thrombolytic therapy. However, there is still a lack of scientific evidence on which methods and how the DOACs' coagulant effect should be monitored. Robust scientific studies with a larger number of participants are needed to define when and how to monitor anticoagulation.

6. Cuker A, Siegal DM, Crowther MA, Garcia DA. Laboratory measurement of the anticoagulant activity of the non-vitamin K oral anticoagulants. J Am Coll Cardiol. 2014;64(11):1128-39.

7. Ofek F, Bar Chaim S, Kronenfeld N, Ziv-Baran T, Berkovitch M. International Normalized Ratio Is Significantly Elevated With Rivaroxaban and Apixaban Drug Therapies: A Retrospective Study. Clin Ther. 2017;39(5):1003-10.

8. Duarte RC, Moreira P, Ferreira C, Figueiredo E, Sternick E, Silveira F et al. Atrial Fibrillation and use of rivaroxaban: performance of the prothrombin time/ INR as a function of time after blood collection. Int J Cardiovasc Sci. 2021; 34(2):116-121.

9. Woodruff AE, Wovkulich MM, Mogle BT, Hassan AK. Association between prothrombin time and bleeding in hospitalized patients receiving rivaroxaban. Am J Health Syst Pharm. 2018;75(22):1783-9.

10. Rottenstreich A, Zacks N, Kleinstern G, Raccah BH, Roth B, Da'as N, et al. Direct-acting oral anticoagulant drug level monitoring in clinical patient management. J Thromb Thrombolysis. 2018;45(4):543-9. 